\title{
Optimizing Statistical Character Recognition Using Evolutionary Strategies to Recognize Aircraft Tail Numbers
}

\author{
Antonio Berlanga \\ Departamento de Informática, EPS, Universidad Carlos III de Madrid, 28911 Leganés, Madrid, Spain \\ Email:aberlan@ia.uc3m.es
}

\begin{abstract}
Juan A. Besada
GPDS, Departamento Señales, Sistemas y Radiocomunicaciónes, ESTIT, Universidad Politécnica de Madrid, 28040 Madrid, Spain Email: besada@grpss.ssr.upm.es
\end{abstract}

\author{
Jesús García Herrero \\ Departamento de Informática, EPS, Universidad Carlos III de Madrid, 28911 Leganés, Madrid, Spain \\ Email: jgherrer@inf.uc3m.es
}

José M. Molina

Departamento de Informática, EPS, Universidad Carlos III de Madrid, 28911 Leganés, Madrid, Spain Email:molina@ia.uc3m.es

\section{Javier I. Portillo}

GPDS, Departamento Señales, Sistemas y Radiocomunicaciónes, ESTIT, Universidad Politécnica de Madrid, 28040 Madrid, Spain Email: javierp@grpss.ssr.upm.es

\author{
José R. Casar \\ GPDS, Departamento Señales, Sistemas y Radiocomunicaciones, ESTIT, Universidad Politécnica de Madrid, 28040 Madrid, Spain \\ Email: jramon@grpss.ssr.upm.es
}

Received 18 December 2002; Revised 13 October 2003; Recommended for Publication by Sergios Theodoridis

\begin{abstract}
The design of statistical classification systems for optical character recognition (OCR) is a cumbersome task. This paper proposes a method using evolutionary strategies (ES) to evolve and upgrade the set of parameters in an OCR system. This OCR is applied to identify the tail number of aircrafts moving on the airport. The proposed approach is discussed and some results are obtained using a benchmark data set. This research demonstrates the successful application of ES to a difficult, noisy, and real-world problem.
\end{abstract}

Keywords and phrases: aircraft recognition, evolutionary strategies for OCR, statistical pattern classifier, image processing.

\section{INTRODUCTION}

We describe the design of an image-based aircraft identification system for an advanced surface movement guidance and control systems (A-SMGCS) $[1,2]$. This work is aimed at implementing some functions of the A-SMGCS concept in Madrid-Barajas international airport, in order to provide aircraft identification. A-SMGCS requires the unambiguous identification of all aircraft and vehicles in the airport movement area. Cameras for this function should be deployed near taxiways and runways, in positions being traversed for all the interest targets, prior to their entrance into the area to be controlled (mainly runways and taxiways). When an aircraft passes in front of the camera (which may be predicted using a tracking system), an image of its tail is captured. An optical character recognition (OCR) applied over aircraft tail number is used to identify aircraft $[3,4]$. In this paper, it is proposed to tune the parameters of the statistical classifier used in the OCR applying an evolutionary computation algorithm. Then, the aircraft identification algorithm is applied and the tracking system is updated with this information. This process is shown in Figure 1. 


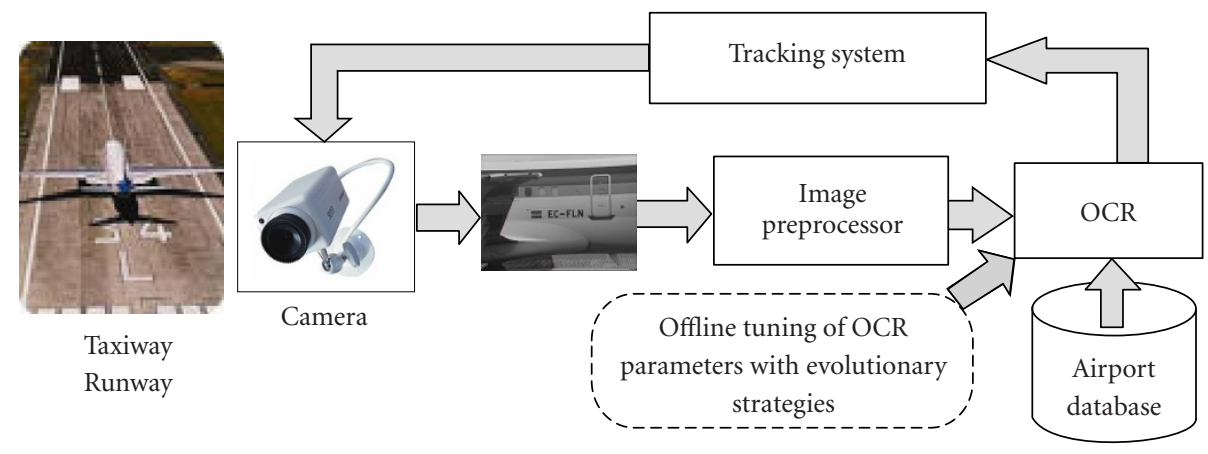

FIGURE 1: Real-time identification procedure of an aircraft.

The aircraft tail number recognition is related to the word recognition problem. Word recognition is done by adding preprocessing and postprocessing steps to the character recognition. Generally, a three-step process is common in character-based methods: character segmentation, character recognition, and contextual postprocessing. The surface airport control maintains a database with aircrafts in runways, taxiways, and terminal areas. This database will be used as the lexicon that specifies the set of allowable words.

Each process in the character-based method has been profusely studied. The character segmentation process could take into account heuristic rules, contour analysis, connected components, and so forth. Previously and related to the election of a classifier, a set of features describing the relevant information of the characters must be selected. A feature, in general terms, is an entity defined by an estimation algorithm. The selected features must be efficiently computable, versatile to represent patterns of the same class, and sensitive to discriminate patterns of different classes. In this domain, the aircraft tail number typography presents a wide variation in the sizes and typesets. Therefore, those features are invariant to transformations on the character needed to be used. A great variety of classification approaches have been studied [5]: neural networks, structural and syntactic classifiers, fuzzy clustering, and statistical [6] and nearest-neighbor classifiers.

In this paper, in order to improve the classification capability, the statistical classifier was fitted by means of an evolutionary strategy (ES). The reason for using this technique is the big size of the space of solutions and the correlations among the parameters of the classifier, requiring an automatic optimization to search appropriate designs. In the evolutionary computation field, the potential correlation among parameters is referred to as epistasis. Problems with little epistasis are easily solved (many gradient descent algorithms can solve them), but highly epistatic problems are difficult and modification of the standard algorithm must be carried out.

Evolutionary Strategies (ES) [7] were developed to solve technical optimization problem. They are based on a metaphor of the theory of evolution proposed by Darwin.
Nowadays, ES are a kind of evolutionary algorithm largely applied to optimization problems in a wide range of technology fields, such as, scheduling, robot controllers, design of electronic circuits, and aerodynamic design.

Fast identification of the aircraft is the main purpose of our system. Therefore, the recognition algorithm must be fast. With this limitation, the simple (but robust) OCR models will be considered. The OCR will be adapted to the characteristics of the tail number typography; we do not intend to develop a general-purpose OCR. The designed OCR will be tuned in order to obtain the best performance for the problem described in this work.

The outline of the paper is as follows. Section 2 describes the features selected to represent characters that are managed by the OCR. In Section 3, the statistical classification model is explained in detail, especially focused in the statistical distance measuring similarity among patterns. The evolutionary learning to adjust the set classifiers parameters is explained in Section 4. The incorporation of the classifier to the whole system and its validation is shown in Section 5. Finally, Section 6 discusses the potential limitations of this approach and presents some conclusions.

\section{AIRCRAFT IDENTIFICATION ALGORITHM}

The tail number recognition can be divided in the following stages.

(i) Frame captures.

(ii) Preprocessing: two preprocessing steps are carried out. The first one searches in the extracted image to identify the region containing the tail number. The second one is applied over this region to isolate single characters.

(iii) Feature extraction: a zoning algorithm is applied to translate each individual character into a vector with the estimated attributes.

(iv) Classification: the pattern with the best matching for each character in the tail number is searched using the statistical classifier described later.

(v) Postprocessing: the airport database is used as a lexicon to solve potential ambiguities in identifications of some characters. 


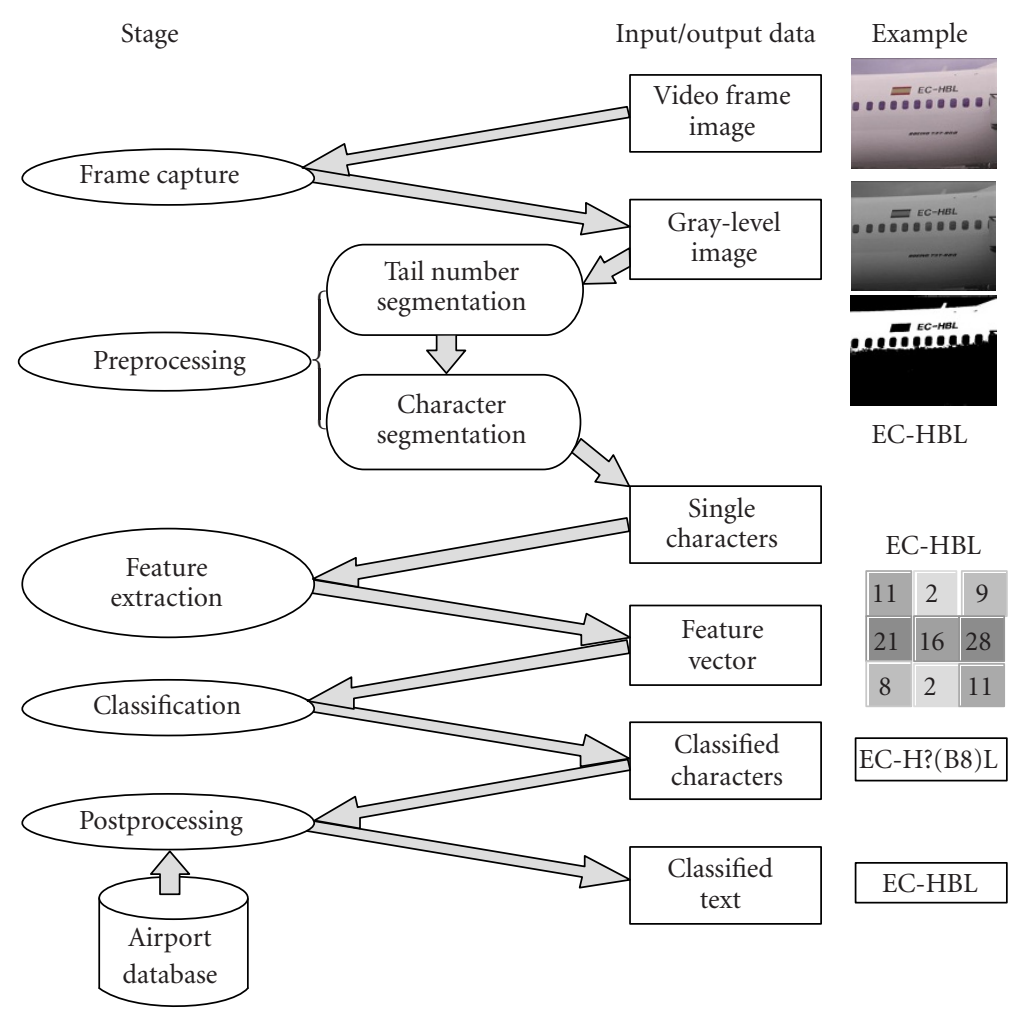

FIGURE 2: General steps for tail number identification.

In Figure 2, previous stages are represented, highlighting the input and output data associated with each stage.

The characteristics of images require robust features that are invariant to changes in size, deformations, brightness, and typography. Thus, the feature extraction procedure must show insensitivity to these changes [8]. Zoning procedures, representing characters as a grid of subimages sampled, show this insensitivity to image conditions on the number of zones selected [9]. The less the number of zones, the more the robustness (but the definition of character is less precise) and more zones show a higher precision (but the behavior with perturbation is less robust).

The proposed procedure uses nine zones $(3 \times 3$ subimages, see Figure 3). This value maintains the tradeoff between computation cost and robustness. On the one hand, an increment in the number of zones demands the application of several algorithms to correct some character deformations as rotation and skew. On the other hand, less than nine zones are insufficient to distinguish characters. Additionally, we will use the number of holes in the character as an additional feature. Figure 3 shows the obtained vector for character " $\mathrm{H}$."

The classifier compares the characters found in the image (its vector representation) with synthetically generated patterns, assigning the joint probability for each pattern ( $p(C, P i)$, where $C$ is the character extracted from the image and $P_{i}$ is the $i$ th pattern). The classifier is optimized using an ES to maximize the posterior probability of assignments.

The comparison between vectors (acquired vector and pattern vector) is performed through a distance function evaluating the similarity between detected characters and ideal patterns. A detailed description of the distance function will be shown in the following section.

Finally, the classifier identifies an aircraft based on the use of the airport database, in which tail numbers for every aircraft in the airport are included. The identification method starts requesting all those tail numbers. We first suppose all tail numbers in the database had the same length $(N)$ and there were only one candidate zone (the correct one) comprising a number of tentative characters equal to that length. In that case, the method to be used would be searching for the maximum joint probability, calculated for each tail number in the database as

$$
P_{\text {tail-number }}=\prod_{i=1}^{N} p\left(C_{i}, P_{\text {tail-number }(i)}\right),
$$

where $P_{\text {tail-number }(i)}$ is the vector representation of the pattern associated to the $i$ th character in the tail number, $C_{i}$ is the vector representation of the tentative character at the $i$ th position in the candidate region, and $p\left(C, P_{j}\right)$ is the aboveexplained probability.

\section{PROBLEM DEFINITION}

We have defined a function to evaluate the similarity between the characters extracted in the image and the ideal patterns representing each alphanumeric symbol. So, it is a classification problem with the patterns represented by real vectors of 

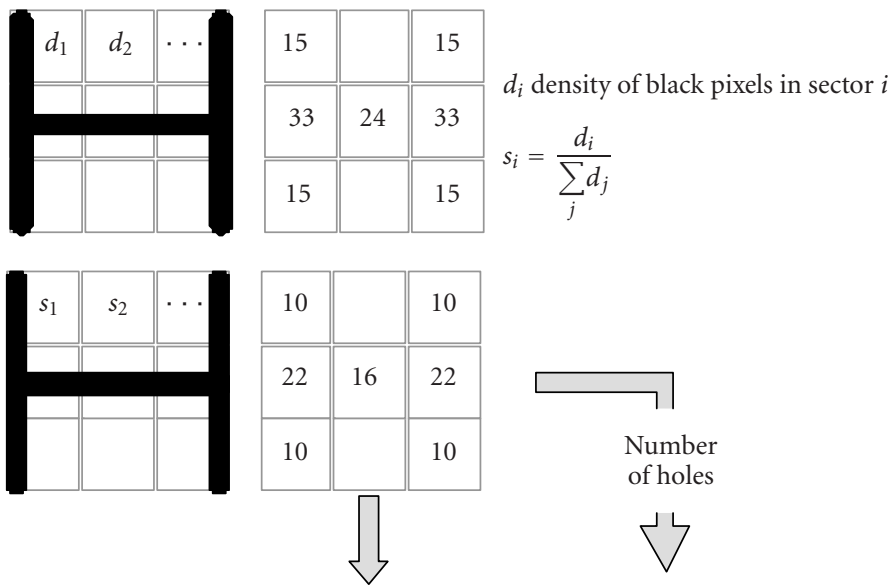

Number of holes

Total density

$(10,0,10,22,16,22,10,0,10,0)$

FIGURE 3: Vector representation including number of holes and total density.

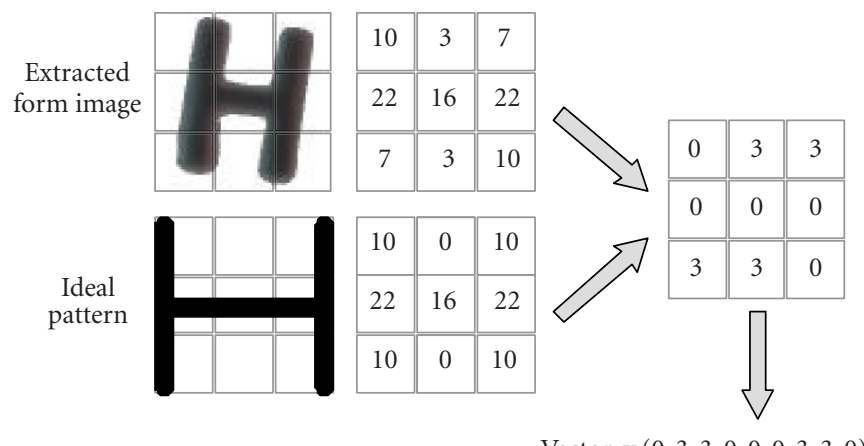

Vector $\mathbf{v}(0,3,3,0,0,0,3,3,0)$

Figure 4: Difference between the ideal pattern " $\mathrm{H}$ " and the extracted form image.

10 components (the average grey levels in the $3 \times 3$ subimage samples and the number of holes), and there are 35 possible classes (possible characters in a given tail number).

The characters are classified using both the distance between the $3 \times 3$ density, $\delta\left(C_{i}, P_{j}\right)$, and the difference in the number of holes, $\delta_{H}\left(C_{i}, P_{j}\right)$, of the tentative characters and the predefined patterns. The density distance is defined as

$$
\begin{gathered}
\delta\left(C_{i}, P_{j}\right)=\mathbf{v}^{T} \Sigma^{-1} \mathbf{v}, \\
\mathbf{v}=\left(\begin{array}{c}
\left|c_{i}^{1}-p_{j}^{1}\right| \\
\vdots \\
\left|c_{i}^{9}-p_{j}^{9}\right|
\end{array}\right), \quad \Sigma^{-1}=\left(\begin{array}{ccc}
\alpha_{1,1} & \cdots & \alpha_{1,9} \\
\vdots & \ddots & \vdots \\
\alpha_{9,1} & \cdots & \alpha_{9,9}
\end{array}\right),
\end{gathered}
$$

with the restriction $\sum_{i=1}^{9} \sum_{j=1}^{9} \alpha_{i j}=1, \alpha_{i j}=\alpha_{j i}$.

Here $\mathbf{v}$ is the difference between ideal pattern $\left(P_{j}\right)$ and detected character $\left(C_{i}\right)$ (see Figure 4 ) and $\Sigma^{-1}$ is a relative weighting matrix. Terms in $\Sigma$ represent covariances in vector components and could be tuned by means of an adjustment process. As an example, Figure 4 illustrates the attribute vectors $\mathbf{v}$ extracted from character " $H$," rotated and skewed, and those predefined for its "ideal" pattern.
Regarding the number of holes, the distance associated is defined as

$$
\delta_{H}\left(C_{i}, P_{j}\right)=\mid C(\text { holes })_{i}-P(\text { holes })_{j} \mid .
$$

A generalized exponential probability density function, $d_{\rho}$, is proposed to describe the variations in the extracted attributes. The joint pdf for differences in attributes between a character $C_{i}$ and its pattern $P_{j}$ is given by the following expression:

$$
d_{\rho}\left(C_{i}\right)=\frac{\exp \left(-\left(\delta\left(C_{i}, P_{j}\right)+\alpha_{H} \delta_{H}\left(C_{i}, P_{j}\right)\right) / \delta_{P}\right)}{\sum_{j=1}^{\text {Patterns }} \exp \left(-\left(\delta\left(C_{i}, P_{j}\right)+\alpha_{H} \delta_{H}\left(C_{i}, P_{j}\right)\right) / \delta_{P}\right)},
$$

where $\alpha_{H}$ is a parameter that weights the contribution of the number of holes to the global distance and that also should be tuned by the ES in the optimization process, and $\delta_{P}$ is a normalization parameter.

The classifier performance could be improved if some regularity in the features of patterns is taken into account. It is assumed that the relevance of every sector is different 


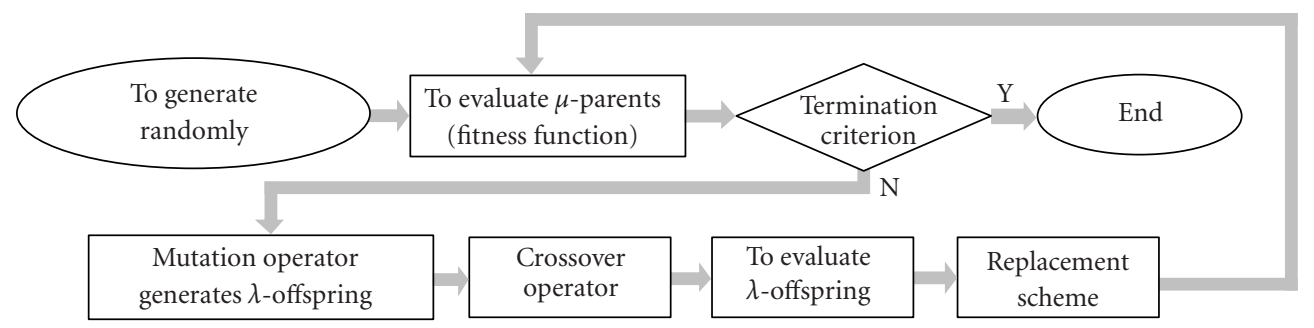

FIGURE 5: General outline of an evolution strategy.

in order to discriminate different patterns. For example, the density of central sector discriminates quite well those patterns with a central hole $(\mathrm{O}, \mathrm{D}, \mathrm{Q}, \mathrm{G}$, and $\mathrm{U})$ from the rest. Conversely, the first sector does not incorporate much information as all patterns have similar density values. Thus, the distance measure may be adjusted through the distance matrix $\Sigma^{-1}$, taking into account this domain information.

However, the design of matrix $\Sigma^{-1}$ can be defined as an optimization problem to globally search the sector weights maximizing the discrimination capacity over all predefined patterns. This optimization process could automatically obtain both the weight terms in attribute distance and the holes-based distance. In the next section, the ES procedure will be applied to optimize this distance measurement between patterns.

\section{LEARNING CLASSIFIER PARAMETERS BY MEANS OF EVOLUTIONARY STRATEGIES}

Evolutionary algorithms combine characteristics of both classifications of classical optimization techniques, volumeoriented and path-oriented methods. Volume-oriented methods (Monte Carlo strategies, clusters algorithms) carry out the searching process scanning the feasible region while path-oriented methods (pattern search, gradient descent algorithms) follow a path in the feasible region. A definition of a restricted search space of the finite volume and the starting point is required to volume-oriented and path-oriented methods, respectively. Evolutionary algorithms characteristics change during the evolutionary process and both exploitation and exploration searches take place. ES are techniques widely used (and more appropriated than genetic algorithm) in real-values optimization problems. Evolutionary computation algorithms offer practical advantages facing difficult optimization problems [10]. These advantages are conceptual simplicity, broad applicability, potentiality to use knowledge and hybridize with other methods, implicit parallelism, robustness to dynamic changes, capability for selfoptimization, and capability to solve problems that have no known solutions.

A general ES is defined as an 8-tuple [7]:

$$
\mathrm{ES}=(I, \Phi, \Omega, \Psi, s, \iota, \mu, \lambda),
$$

where $I=(\vec{x}, \vec{\sigma}, \vec{\alpha})=\mathcal{R}^{n} \times \mathcal{R}_{+}^{n_{\sigma}} \times[-\pi, \pi]^{n_{\alpha}}$ is the space of individuals, $n_{\alpha} \in\{1, \ldots, n\}$ and $n_{\alpha} \in\left\{0,\left(2 n-n_{\alpha}\right)\left(n_{\sigma}-\right.\right.$
1)/2\}, $\Phi: I \rightarrow \mathcal{R}=f$ is the fitness function, and $\Omega=$ $\left\{m_{\left\{\tau, \tau^{\prime}, \beta\right\}}: I^{\lambda} \rightarrow I^{\lambda}\right\} \cup\left\{r_{\{r, r \sigma, r \alpha\}}: I^{\mu} \rightarrow I^{\lambda}\right\}$ are the genetic operators, mutation and crossover operators. $\Psi(P)=$ $s\left(P \cup m_{\left\{\tau, \tau^{\prime}, \beta\right\}}\left(r_{\{r x, r \sigma, r \alpha\}}(P)\right)\right)$ is the process to generate a new set of individuals, $s$ is the selection operator, and $\iota$ is the termination criterion.

In this work, the definition of the individual has been simplified: the rotation angles $n_{\alpha}$ have not been taken into account, $n_{\alpha}=0$.

The mutation operator generates new individuals as follows:

$$
\begin{gathered}
\sigma_{i}^{\prime}=\sigma_{i} \cdot \exp \left(\tau^{\prime} \cdot N(0,1)+\tau \cdot N_{i}(0,1)\right), \\
\vec{x}^{\prime}=\vec{x}+\sigma_{i}^{\prime} \cdot \vec{N}(\overrightarrow{0}, 1) .
\end{gathered}
$$

In Figure 5, the general outline of ES is showed.

ES has several formulations, but the most common form is $(\mu, \lambda)$-ES, where $\lambda>\mu=1,(\mu, \lambda)$ means that $\mu$-parents generate $\lambda$-offspring through crossover and mutation in each generation. The best $\mu$ offsprings are selected deterministically from the $\lambda$ offspring and replace the current parents. ES considers that strategy parameters, which roughly define the size of mutations, are controlled by a "self-adaptive" property of their own. An extension of the selection scheme is the use of elitism; this formulation is called $(\mu+\lambda)$-ES. In each generation, the best $\mu$-offspring of the set $\mu$-parents and $\lambda$ offspring replace the current parents. Thus, the best solutions are maintained through generation. The computational cost of $(\mu, \lambda)$-ES and $(\mu+\lambda)$-ES formulation is the same.

The type of crossover used in this work is the discrete crossover and the two standard types of ES replacement schemes, $(\mu+\lambda)$-ES and $(\mu, \lambda)$-ES, were used to select the individual to the next generation.

\subsection{Codification of OCR model}

In this identification model, the parameters $\alpha_{H}$ and $\alpha_{i j}$ values in $\Sigma$ may be correlated, thus, the global optimization procedure must simultaneously adjust all of them. In this case, the global optimization problem has a unique restriction; the elements of distance matrix are normalized to 1 , see (2). This restriction is included in the codification and all individuals are processed to become feasible ones. Then, in spite of this restriction, the solutions space does not have infeasible regions. In this way, the problem has a multimodal solution space and one solution could have several representations. 
In this work, an individual is codified as a 46dimensional real vector as follows: $\vec{x}=\left(\Sigma^{-1}, \alpha_{H}\right)=\left(\alpha_{1,1}\right.$, $\left.\alpha_{1,2}, \ldots, \alpha_{9,9}, \alpha_{H}\right)$, the distance matrix has been taken symmetrical, $\alpha_{i, j}=\alpha_{j, i}$.

The calculation of fitness function is presented now, assuming the exponential pdf presented in Section 4 to model variations in pattern attributes. The design criterion was to maximize the probability of correctly classifying a pattern compared with itself and with the rest of patterns, taking as goal the worst case. The effect of errors in the measurement of the number of holes is considered in (8), taking no errors in the number of holes, and in (10), when errors are considered. Besides, a certain probability of error in classifying a pattern with itself is included, with term $d_{\text {noise }}$ representing spurious variations in the features. The associated probabilities for these distances (distance to the own pattern in comparison to distance to the rest of patterns) are computed in (9) and (11), with the appropriate normalization. Equation (12) is the probability, with and without errors, in the number of holes and (13) is the lower probability of right classification among all patterns, representing so the worst case:

$$
\begin{aligned}
& d_{\rho}\left(C_{i}\right)=\left\{\begin{array}{l}
\sum_{j=1}^{n} \exp \left(-\frac{\delta\left(C_{i}, P_{j}\right)+\alpha_{H} \delta_{H}\left(C_{i}, P_{j}\right)}{\delta_{P}}\right), \quad i \neq j, \\
\sum_{j=1}^{n} \exp \left(-\frac{d_{\text {noise }}}{\delta_{P}}\right), \quad i=j,
\end{array}\right. \\
& \rho_{i}=\frac{\exp \left(-d_{\text {noise }} / \delta_{P}\right)}{d_{\rho}\left(C_{i}\right)}, \\
& d_{\sigma}\left(C_{i}\right)= \begin{cases}\sum_{j=1}^{n} \exp \left(-\frac{\delta\left(C_{i}, P_{j}\right)}{\delta_{P}}\right), & i \neq j, \\
\sum_{j=1}^{n} \exp \left(-\frac{d_{\text {noise }}+\alpha_{H}}{\delta_{P}}\right), & i=j,\end{cases} \\
& \sigma_{i}=\frac{\exp \left(-\left(d_{\text {noise }}+\alpha_{H}\right) / \delta_{P}\right)}{d_{\sigma}\left(C_{i}\right)}, \\
& P_{i}=\left(1-p_{H}\right) \rho_{i}+p_{H} \sigma_{i}, \\
& f=\min \left[P_{i}\right]_{i=1}^{n} \text {. }
\end{aligned}
$$

The parameter $d_{\text {noise }}$ represents so the average distance of a pattern with itself due to noise, and in this work was fixed to $3 \%$; $p_{H}$, the error probability in the estimation of number of holes in a character, has been fixed to 0.05 . The value of $P_{i}$ is the discrimination probability.

The optimization is guided in order to achieve an improvement of the discrimination power. The definition of the discrimination probability allows maximizing the difference between the probability to recognize a pattern with itself, and the most similar pattern to it. A slight modification still must be done in order to apply the ES methodology. The ES is defined to minimize a quality measurement, the fitness function. Thus, the goal function is defined as the complementary probability, in order to minimize the probability of error as follows:

$$
f^{\prime}=1-f
$$

\section{EXPERIMENTS}

In this section, the optimization process performed to adjust the OCR parameters is described first and then we summarize the validation carried out with the set of available test images. Regarding this validation, the segmentation phase was successful for $100 \%$ of available images with tail numbers, and $100 \%$ of characters contained. So, input data to OCR system were the correct subimages representing isolated characters.

\subsection{Evolution strategies to optimize character identification}

The application of ES in order to tune the OCR parameters was previously used by other authors [11]. In this work, it has been applied as an optimization method of probabilistic detection parameters to obtain the distance matrix $\left(\alpha_{i j}\right)$ and $\alpha_{H}$ (that weights the difference in the number of holes).

Following the method suggested by Schwefel [12], that assures the convergence of ES to a set of solutions with the same fitness value, the number of different runs must be $N^{\prime} \approx 90$. The parameters of the ES are summarized in Table 1 .

Two different ES procedures (with/without elitism) were performed using the parameters summarized in Table 1. In Figure 6, the average of best fitness value (for 100 runs) in each generation step was plotted. The standard deviation is drawn as vertical lines.

The convergence of learning process is quite fair, the process always converges to solutions with the same fitness value. As can be appreciated, the shapes of the evolutionary process are slightly different (as expected). The $(\mu+\lambda)$-ES (bold line in Figure 6), using an elitism selection procedure, converges in 200 generations, while $(\mu, \lambda)$-ES (thin line) requires 800 generations to be near the final fitness obtained by $(\mu+\lambda)$ ES. The analysis of these results seems to conclude that using a $(\mu+\lambda)$-ES achieves better results (at least in execution time) than using a $(\mu, \lambda)$-ES. This conclusion may be premature, since results depend on problem specifications. When the problem has a unique solution (or a small region of solutions) and fitness functions are smooth, the elitism selection procedure, that performs a depth search, is highly recommend. When the problem specification cannot assure the above premises about the region of solutions and fitness function, a nonelitism procedure, that performs a breath search, allows obtaining better solutions with a worse time performance.

A low value of standard deviation in the fitness function of population (see Figure 6) at the end of the training process indicates that solutions (100 vectors) have a similar fitness value. Low fitness value proves the convergence in terms of fitness, but not that all solutions represent a unique solution. A clustering algorithm could carry out the evaluation of this fact.

Let $x^{i}\left(x_{1}^{i}, x_{2}^{i}, x_{3}^{i}, \ldots, x_{46}^{i}\right)$ be a solution, $\left\{x^{i}\right\}_{i=0,100}$ the set of solutions, and assume that each parameter follows a normal distribution. In this work, we used CLUTO v2.0 [13], a freely distributed software package for clustering datasets. 
TABLE 1: Setting of exogenous parameters of the ES.

\begin{tabular}{ll}
\hline Parameter & Value \\
\hline Initial standard deviations $\sigma_{i}(0)$ & Randomly generated in range $[0.0,3.0]$ \\
Number of rotation angles $n_{\alpha}$ & 0 \\
Parent population size $\mu$ & 10 \\
Offspring population size $\lambda$ & 80 \\
Termination criterion $\iota$ & Number of generation step $=1000$ \\
\hline
\end{tabular}

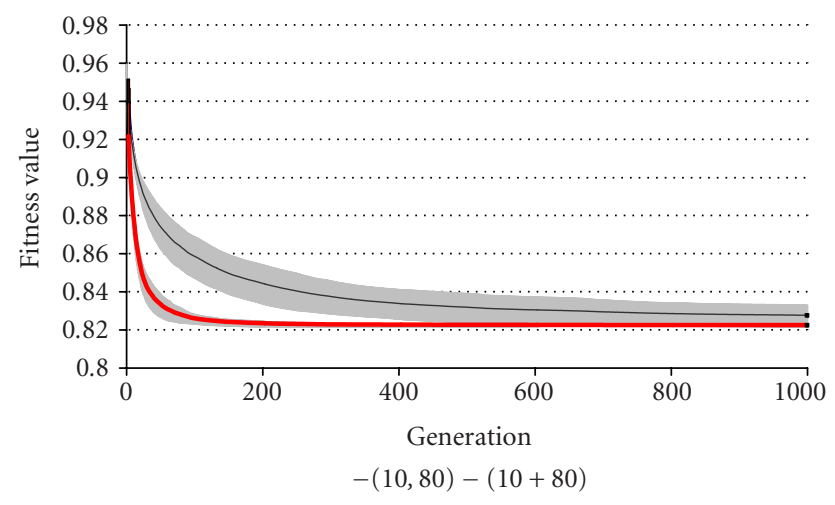

Figure 6: Evolution of best fitness value.

The clusters are obtained applying a graph-partitioning clustering algorithm that computes the similarity between objects, inversely proportional to the Euclidean distance. Clustering criterion function to be used in finding the minimum clusters partition was evaluated. The analysis of CLUTO results validated the hypothesis that lineal solutions, evolved with $(\mu, \lambda)$-ES and $(\mu+\lambda)$-ES, are in just one cluster each. Thus, the average of solutions (the centroid of the cluster) is enough to represent the 100 different solutions. In Tables 2 and 3 the averaged parameters are presented.

The fittest result shows nonzero values in the diagonal of the distance matrix, that is, negligible correlation exists between different sectors. The distance that maximizes the interclasses discrimination is achieved taking only into account the differences in common sectors without considering intersector terms. It can be noticed, as the result obtained for the weight corresponding to the first sector suggests, that this must not be taken into account in the calculation of distance among characters and patterns.

\subsection{Validation of OCR's performance}

In order to validate the OCR performance, 115 images were used from a set of one thousand recorded images taken from 60 aircrafts. The discarded images (unresolved for the human eye) could not be used in the identification process due to their low quality. The images comprise the tail number and were taken from several distances and perspectives. Three types of images have been taken per aircraft (in the same proportion). (i) Near-distance images, where the tail number is centred in the image, and it is taken orthogonal to the aircraft.

(ii) Medium-distance images, where the tail number appears with other objects (e.g., windows, flags, etc.). Furthermore, the tail number appears distorted by the effect of the aircraft fuselage curvature.

(iii) Long-distance images, where the tail number is confused with the set of objects that appear in the image (e.g., wings, motor, soil, sky, staircases, etc.).

In Figure 7, there are some sample images used in this work, where the variability due to geometric transformations, intensity and sizes can be observed. The image regions representing characters may suffer from spatial transformations (because of the relative position of the camera), variability of grey-level characteristics (because of different atmospheric conditions or color of the character), and variations in the letters size along different aircraft. Thus, for example, considering these images, the tail numbers "ECDLH" and "EC-FLN" sizes are 526 by 134 pixels and 230 by 45 pixels, respectively.

The distance matrix obtained with the ES and a simple one used as reference were incorporated to compare performances of different classifiers. All components of the reference matrix were set to $1 / 81$, that is, a classifier without information about the features of characters (Euclidean distance) was considered for comparison. The classifier was applied over the 115 test images and the postprocessing step selected the tail number of the airport database, with maximum joint probability. As mentioned above, the segmentation phase 
TABLE 2: Distance matrix $\Sigma^{-1}$ and $\alpha_{H}$ averaged in 100 runs of $(\mu, \lambda)$-ES. Values below $1 \mathrm{E}-3$ are set to zero.

\begin{tabular}{|c|c|c|c|c|c|c|c|c|c|}
\hline \multirow{9}{*}{$\Sigma^{-1}$} & 0 & 0 & 0 & 0 & 0 & 0 & 0 & 0 & 0 \\
\hline & 0 & 2,9E-01 & 0 & 0 & 0 & 0 & 0 & $1,0 \mathrm{E}-03$ & $4,8 \mathrm{E}-03$ \\
\hline & 0 & 0 & $9,6 \mathrm{E}-03$ & 0 & 0 & 0 & 0 & 0 & 0 \\
\hline & 0 & 0 & 0 & $6,0 \mathrm{E}-02$ & 0 & 0 & 0 & 0 & 0 \\
\hline & 0 & 0 & 0 & 0 & $9,5 \mathrm{E}-02$ & 0 & $1,4 \mathrm{E}-03$ & $1,5 \mathrm{E}-03$ & 0 \\
\hline & 0 & 0 & 0 & 0 & 0 & $6,2 \mathrm{E}-02$ & $1,5 \mathrm{E}-03$ & 0 & 0 \\
\hline & 0 & 0 & 0 & 0 & $1,4 \mathrm{E}-03$ & $1,5 \mathrm{E}-03$ & 2,7E-01 & 0 & 0 \\
\hline & 0 & $1,0 \mathrm{E}-03$ & 0 & 0 & $1,5 \mathrm{E}-03$ & 0 & 0 & $1,1 \mathrm{E}-01$ & 0 \\
\hline & 0 & $4,8 \mathrm{E}-03$ & 0 & 0 & 0 & 0 & 0 & 0 & $6,0 \mathrm{E}-02$ \\
\hline$\alpha_{H}$ & 342,7 & & & & & & & & \\
\hline
\end{tabular}

TABLE 3: Distance matrix $\Sigma^{-1}$ and $\alpha_{H}$ averaged in 100 runs of $(\mu+\lambda)$-ES. Values below $1 \mathrm{E}-3$ are set to zero.

\begin{tabular}{|c|c|c|c|c|c|c|c|c|c|}
\hline \multirow{9}{*}{$\Sigma^{-1}$} & 0 & 0 & 0 & 0 & 0 & 0 & 0 & 0 & 0 \\
\hline & 0 & $3,2 \mathrm{E}-01$ & 0 & 0 & 0 & 0 & 0 & 0 & 0 \\
\hline & 0 & 0 & $7,6 \mathrm{E}-03$ & 0 & 0 & 0 & 0 & 0 & 0 \\
\hline & 0 & 0 & 0 & $5,2 \mathrm{E}-02$ & 0 & 0 & 0 & 0 & 0 \\
\hline & 0 & 0 & 0 & 0 & $9,0 \mathrm{E}-02$ & 0 & 0 & 0 & 0 \\
\hline & 0 & 0 & 0 & 0 & 0 & $6,9 \mathrm{E}-02$ & 0 & 0 & 0 \\
\hline & 0 & 0 & 0 & 0 & 0 & 0 & 2,7E-01 & 0 & 0 \\
\hline & 0 & 0 & 0 & 0 & 0 & 0 & 0 & 1,2E-01 & 0 \\
\hline & 0 & 0 & 0 & 0 & 0 & 0 & 0 & 0 & $7,0 \mathrm{E}-02$ \\
\hline
\end{tabular}
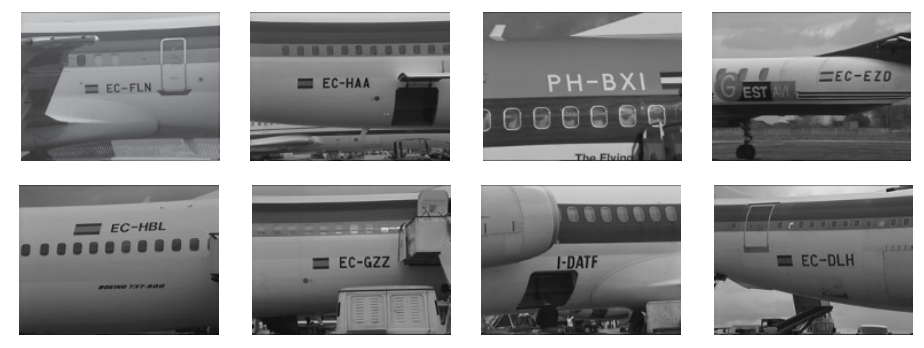

Figure 7: Several test images. was successful for all test images, providing the OCR with the correct image regions in all the cases.

In Figure 8, the validation results are presented including the histogram of the maximum joint probability.

Therefore, the best performance was achieved with the classifier that incorporates the distance matrix evolved by means of the $(10+80)$-ES; its histogram is displaced to higher values of maximum joint probability. The mean improve in the tail number recognition task is summarized in Table 4.

In this problem, to obtain a better character classifier will induce achieving a better "word" classifier too. An identification improvement of $1.6 \%$ per image justifies the application of an optimization process, in this case, due to the characteristic of the space problem, applying the ES paradigm.
In our tests, all tail numbers were correctly identified using the airport database, as far as the maximum probability corresponds to the actual tail number.

\section{CONCLUSIONS}

In this work, an ES has been applied to optimize the set of parameters of an OCR. The method was chosen because of its easy implementation and good tradeoff between complexity and performances. The identification of tail number was improved using the correlation of different sectors, to identify characters, in the statistical classifier. Furthermore, a hierarchical discrimination of characters, adding some other character features, as number of joint points, will surely enhance 


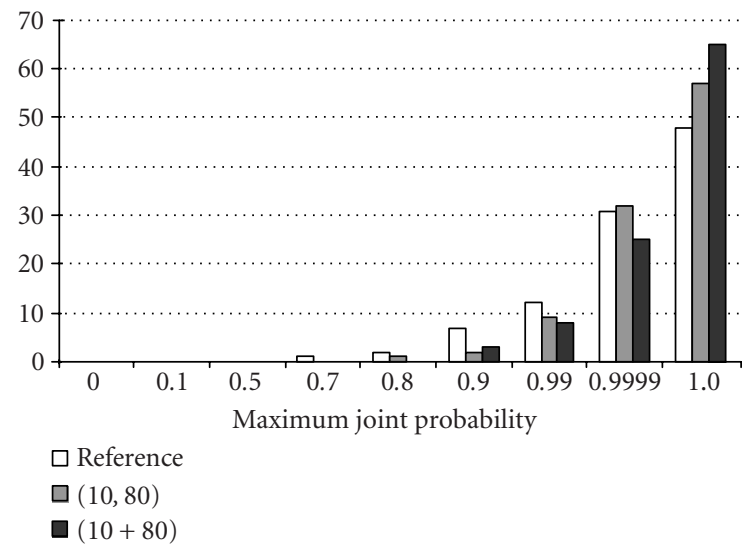

FIGURE 8: Histogram of OCR results.

TABle 4: Percentage of improvement per image of the maximum joint probability calculates between classifiers.

\begin{tabular}{lc}
\hline Classifiers & Improvement \\
\hline$(10+80)$-ES versus $(10,80)$-ES & $0.1 \%$ \\
$(10+80)$-ES versus reference & $1.6 \%$ \\
$(10,80)$-ES versus reference & $1.5 \%$ \\
\hline
\end{tabular}

the global performance of the tail number identification. We have tested the behavior of the described system with 115 real images taken in Madrid/Barajas Airport. They were recorded from 60 different tail numbers, viewed from different positions. Results show that our system is quite robust although its discriminating capability would be able to improve.

\section{ACKNOWLEDGMENTS}

The authors recognize the support provided by AENA (Aeropuertos Españoles y Navegación Aérea) with special thanks to Angeles Varona and Germán Gonzalez for their help. This work has been funded by the Spanish CICYT contract TIC2002-04491-C01/02.

\section{REFERENCES}

[1] "The Future Airport Surface Movement Safety, Guidance, and Control System: A Vision for Transition into the 21st Century US Department of Transportation," Federal Aviation Administration, Washington, DC, USA, 1993.

[2] "Manual on Advanced Surface Movement and Control Systems (ASMGCS)," Draft Volume 1: Operational Requirements. Draft Version 04. 08/24/2000.

[3] Y. Cui and Q. Huang, "Extracting characters of license plates from video sequences," Machine Vision and Applications, vol. 10, no. 5/6, pp. 308-320, 1998.

[4] J. A. Besada, J. Portillo, J. Garcia, and J. M. Molina, "Imagebased automatic surveillance for airport surface," in Fourth International Conference on Information Fusion, Montreal, Canada, August 2001.

[5] J. R. Parker, Algorithms for Image Processing and Computer Vision, John Wiley \& Sons, New York, NY, USA, 1996.
[6] S. M. Kay, Fundamentals of Statistical Signal Processing: Detection Theory, Prentice Hall, Englewood-Cliffs, NJ, USA, 1998.

[7] T. Bäck, Evolutionary Algorithms in Theory and Practice, Clarendon Press, Oxford University Press, New York, NY, USA, 1996.

[8] Ø. D. Trier, A. K. Jain, and T. Taxt, "Feature extraction methods for character recognition-A survey," Pattern Recognition, vol. 29, no. 4, pp. 641-662, 1996.

[9] M. Bokser, "Omnidocument technologies," Proceedings of the IEEE, vol. 80, no. 7, pp. 1066-1078, 1992.

[10] D. B. Fogel, "The advantages of evolutionary computation," in Proc. BioComputing and Emergent Computation, D. Lundh, B. Olsson, and A. Narayanan, Eds., pp. 1-11, World Scientific, Skövde, Singapore, July 1997.

[11] G. Adorni, F. Bergenti, and S. Cagnoni, "Vehicle license plate recognition by means of cellular automata," in Proc. IEEE International Conference on Intelligent Vehicles, pp. 689-693, Stuttgart, Germany, October 1998.

[12] H.-P. Schwefel, "Evolutionary learning optimum-seeking on parallel computer architectures," in Proceedings of the International Symposium on Systems Analysis and Simulation 1988, I: Theory and Foundations, A. Sydow, S. G. Tzafestas, and R. Vichnevetsky, Eds., vol. 46, pp. 217-225, Akademie-Verlag, Berlin, Germany, September 1988.

[13] CLUTO v2.0, George Karypis. Copyrighted by the Regents of the University of Minnesota, http://www.cs.umn.edu/ $\sim$ karypis/cluto.

Antonio Berlanga received his B.S. degree in physics from Universidad Autónoma, Madrid, Spain, in 1995, and his Ph.D. degree in computer engineering from Universidad Carlos III de Madrid in 2000. Since 2002, he has been there as an Assistant Professor of automata theory and programming language translation. His main research topics are evolutionary computation applications and network optimization using soft computing.

Juan A. Besada received his Master's degree in telecommunication engineering from Universidad Politécnica de Madrid (UPM) in 1996 and his Ph.D. degree from the same university in 2001. He has worked in the Signal and Data Processing Group of the same university since 1995, participating in several national and European projects related to air traffic control. He is currently an Associate Professor at Universidad

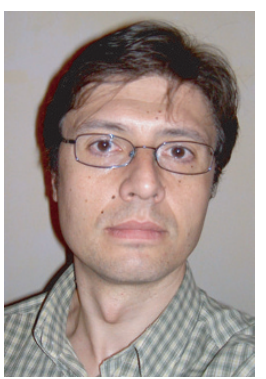
Politécnica de Madrid (UPM). His main interests are air traffic control, navigation, and data fusion.

Jesús García Herrero received his Master's degree in telecommunication engineering from Universidad Politécnica de Madrid (UPM) in 1996 and his Ph.D. degree from the same university in 2001. He has been working as a Lecturer at the Department of Computer Science, Universidad Carlos III de Madrid, since 2000. There, he is also integrated in the Systems, Complex and Adaptive Laboratory, involved in artificial intel-

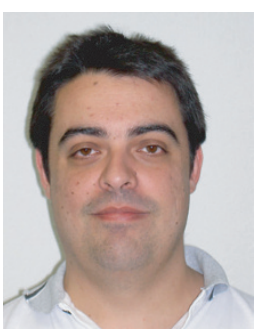
ligence applications. His main interests are radar data processing,

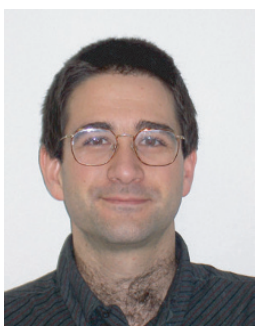


navigation, and air traffic management, with special stress on data fusion for airport environments. He has also worked in the Signal and Data Processing Group of UPM since 1995, participating in several national and European research projects related to air traffic control.

José M. Molina received his Master's degree in telecommunication engineering from Universidad Politécnica de Madrid (UPM) in 1993 and his Ph.D. degree from the same university in 1997. He is an Associate Professor at Universidad Carlos III de Madrid. His current research focuses on the application of soft computing techniques (NN, evolutionary computation, fuzzy logic, and multiagent systems) to radar data process-

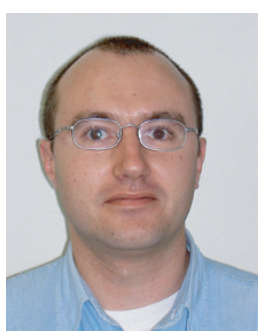
ing, navigation, and air traffic management. He joined the Computer Science Department of Universidad Carlos III de Madrid in 1993, being enrolled in the Systems, Complex, and Adaptive Laboratory. He has also worked in the Signal and Data Processing Group of UPM since 1992, participating in several national and European projects related to air traffic control. He is the author of up to 10 journal papers and 70 conference papers.

Javier I. Portillo obtained his B.S. and Ph.D. degrees in telecommunication engineering in 1985 and 1991, respectively, both from the Polytechnic University of Madrid. Currently, he is Professor in the Telecommunication Engineering School of the Polytechnic University of Madrid, in the Signal, System, and Radiocommunication Department. His research interests are image processing, computer vision and simulation,

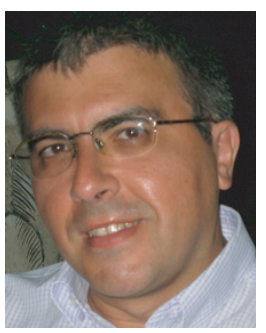
and the application of the preceding techniques to air traffic control. He is author or coauthor of many research papers and technical reports and he has worked in or managed more than 30 research public projects and private projects with enterprises.

José R. Casar received his graduate degree in telecommunications engineering in 1981 and his Ph.D. degree in 1983 from the Universidad Politécnica de Madrid (UPM). He is a Full Professor in the Department of Signals, Systems, and Radiocommunications of UPM. At the present time he is an Adjunct to the Rector for Strategic Programs and Head of the Data Processing and Simulation Group at the same university. His re-

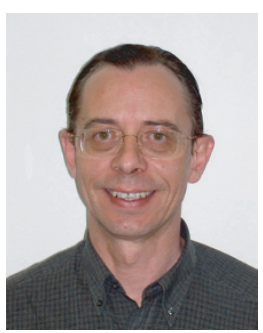
search interests include radar technologies, signal and data processing, multisensory fusion, and image analysis both for civil and defence applications. During 1993, he was a Vice Dean for Studies and Research at the Telecommunications Engineering School of UPM. During 1995, he was a Deputy Vice President for Research at UPM and from 1996 to 2000 he was a Vice President for Research at UPM. 